\title{
Estudo da reologia de pastas cimentícias contendo resíduo de tijolo cerâmico moído e metacaulim
}

\author{
Study of the rheology of cement pastes \\ containing ceramic brick wastes end \\ metakaolin
}

\author{
João Sérgio Filho ${ }^{1}$, Kássia Sinhorelli ${ }^{2}$, \\ Guilherme Medeiros ${ }^{1}$, Aline Azeredo ${ }^{3}$,
} Givanildo Azeredo 4

\footnotetext{
${ }^{1}$ Universidade Federal da Paraíba, Campus I, Cidade Universitária, s/n, João Pessoa, PB, Brasil.

${ }^{2}$ Universidade Federal da Paraíba, Campus I, Cidade Universitária, s/n, João Pessoa, PB, Brasil.

${ }^{3}$ Universidade Federal de Campina Grande, Rua Aprígio Veloso, 882, Campina Grande, PB, Brasil.

${ }^{4}$ Universidade Federal da Paraíba, Campus I, Cidade Universitária, s/n, João Pessoa, PB, Brasil. e-mail: joaosergio52@gmail.com, kassiasinhorelli@hotmail.com, guilhermealef@hotmail.com, alinefnobrega@hotmail.com, givanildoazeredo@hotmail.com
}

\section{RESUMO}

A indústria da construção tem procurado o uso de soluções sustentáveis, uma delas é a incorporação de resíduos como materiais de construção. Para isso, estudos vêm sendo desenvolvidos para analisar a viabilidade da incorporação desses resíduos em argamassas e concretos, visto que essas inserções alteram as propriedades dos materiais tanto no estado endurecido quanto fresco. A análise de materiais como pastas, argamassas ou concretos em seu estado fresco está diretamente ligada ao estudo de uma simulação das solicitações que essas podem sofrer durante sua mistura, transporte e aplicação. Os materiais constituintes e a relação água/aglomerante têm um papel fundamental nas propriedades das pastas. Características como a finura do aglomerante, a forma da partícula e a origem mineralógica podem afetar diretamente a sua trabalhabilidade. Para o entendimento do comportamento reológico de pastas cimentícias no estado fresco, várias técnicas podem ser utilizadas como a reometria de fluxo e oscilação, o método do espalhamento pelo mini-cone, escoamento pelo Funil de Marsh, método do Squeeze flow e o Vane test. Neste trabalho estudouse o comportamento reológico de diferentes pastas cimentícias preparadas com o cimento Portland CP V-ARI RS e adição de metacaulim (MC) e adição de resíduo de tijolo cerâmico moído (RTM). As pastas foram analisadas para diferentes tempos de hidratação e também diferentes relações água/aglomerante. O estudo se deu através de medições de parâmetros reológicos como tensão de escoamento e viscosidade, usando um reômetro rotacional de placas paralelas. Os resultados mostraram que o RTM provocou uma diminuição na tensão de escoamento inicial quando comparado com o uso do MC, o que pode contribuir para uma mistura com maior facilidade de manuseio, ou seja, menos viscosa.

Palavras-chave: Reologia. Pozolana. Resíduo Cerâmico Moído. Metacaulim. Pastas.

\section{ABSTRACT}

The construction industry has sought the use of sustainable solutions, one of which is the incorporation of waste as building materials. For this, studies have been developed to analyze the viability of the incorporation these residues in mortar and concrete, since these inserts modify the properties of the materials both in the hardened and fresh state. The analysis of materials such as pastes, mortars or concretes in their fresh state is directly linked to the study of a simulation of the requests that they may suffer during their mixing, transportation and application. The constituent materials and the water / binder ratio have a fundamental role in the properties of the pastes. Characteristics of the materials such as the fineness of the binder, the shape of the particle and the mineralogical origin can directly affect its workability. For the understanding of the rheological behavior of cement pastes in the fresh state, several techniques can be used such as flow and oscillation rheometry, the mini-cone spreading method, Marsh funnel flow, Squeeze flow method and the Vane test. In 
this work the rheological behavior of different cementitious pastes prepared with Portland cement CP V-ARI RS and addition of metakaolin (MK) end ceramic brick wastes (CBW) was studied. The pastes were analyzed for different hydration times and water/binder ratios. The experiment was carried out through measurements of rheological parameters such as flow stress and viscosity, using a parallel plate rotational rheometer. The results showed that the CBW caused a decrease in the initial flow stress when compared to the use of MK, which may contribute to a mixture with easier handling, that is, less viscous.

Keywords: Rheology. Pozzolan. Ceramic waste. Metakaolin. Pastes.

\section{INTRODUÇÃO}

Para entender a influência e o comportamento de argamassas e concretos no estado fresco é necessário conhecer os parâmetros reológicos do material através do estudo da reologia de sua pasta, visto que, estas propriedades estão diretamente relacionadas com a mesma [1]. A reologia é o ramo da ciência que estuda o fluxo e a deformação da matéria, avaliando a relação entre a tensão de cisalhamento e a deformação ao longo do tempo [2]. A deformação de um corpo pode ser dividida em dois tipos: espontânea e reversível, conhecida como elasticidade, e irreversível, chamada de escoamento [3].

Vários métodos podem ser utilizados para se estudar o comportamento reológico dos materiais, tais como: reometria de fluxo, ensaio oscilatório (varredura de tempo e varredura de deformação) e ensaio de palheta ou Vane test [4-9]. Métodos como o espalhamento pelo mini-cone e escoamento pelo funil de Marsh também são muito utilizados. Todos esses métodos, com exceção da reometria, fornecem dados das características reológicas como a tensão de escoamento e a consistência de forma indireta.

De modo geral, os materiais se classificam como sólidos, líquidos e gasosos, mas a maioria dos corpos reais não se comportam nem como sólidos e nem como líquidos ideais. Vários materiais utilizados na atualidade apresentam comportamento reológico entre o líquido e o sólido ideal, ou seja, são parcialmente viscosos e elásticos, chamados de viscoelásticos [3]. Dentre eles estão os materiais a base de cimento, que têm comportamento viscoelástico conforme STRUBLE e LEI [10].

Da literatura é conhecido que os fluidos se classificam em não-Newtoninano e Newtonianos. Ainda é possível classificar os fluidos não-Newtonianos como Binghamiano, pseudoplásticos e dilatantes. O comportamento destes é definido pela relação entre tensão de cisalhamento e taxa de cisalhamento.

Os fluidos Newtonianos são fluidos onde a relação entre tensão cisalhante e a taxa de cisalhamento é constante, ou seja, sua viscosidade é única, constante. Esse tipo de fluido é expresso pela equação 1:

$$
\tau=\mu \gamma
$$

onde, $\tau$ é a tensão de cisalhamento, $\mu$ é a viscosidade e $\gamma$ a taxa de cisalhamento.

No caso dos fluidos não-Newtonianos essa relação entre tensão e taxa de cisalhamento não é constante, apresentando uma viscosidade variável. As argamassas e concretos são classificados como fluidos nãoNewtonianos. Vários estudos sobre reologia de materiais cimentícios têm indicado que o comportamento dessas misturas segue de forma aproximada ao modelo de Bingham [2, 10 e 11].

VANCE et al. [12] mostraram a relação não-newtoniana entre a tensão de cisalhamento e taxa de cisalhamento, descrevendo que o modelo de Bingham é bastante empregado para a definição reológica das pastas de cimento e que um bom ajuste linear entre estas é conseguido com um intervalo de taxa de cisalhamento entre 10 e $100 / \mathrm{s}$

O modelo de Bingham define o fluido através de dois parâmetros: a tensão de escoamento inicial $\left(\tau_{0}\right)$ e a viscosidade, como mostrado na equação 2 .

$$
\tau=\tau_{0}+\mu \gamma
$$

FERRARIS et al. [13] mostraram que para a determinação dos parâmetros de Bingham há duas possibilidades:

I. A tensão aplicada ao material é elevada lentamente e a taxa de cisalhamento é medida. A mistura começa a escoar quando é aplicada uma tensão alta o suficiente para que isso ocorra, o ponto em que isso acontece é a tensão de escoamento e a inclinação da reta acima desse ponto é a viscosidade plástica, como mostrado na equação 2 . 
II. A mistura é cisalhada a uma alta taxa de cisalhamento antes do teste, depois, essa taxa é diminuída gradualmente e a tensão é medida. A relação entre a tensão e a taxa de cisalhamento é plotada, a tensão de escoamento é definida onde o ponto de interseção dessa taxa é zero, e a inclinação da curva acima deste ponto é a viscosidade plástica.

Além do modelo de Bingham, FERRARIS et al. [13] mostraram que para o concreto auto adensável (CAA), por exemplo, o modelo que melhor descreve o comportamento reológico desse material é o de Herschel-Bulkley (HB). Este modelo também é conhecido como fluido de potência com limite de escoamento e é definido por três parâmetros. A equação 3 é quem o define:

$\tau=\tau_{0}+k(\gamma)^{n}$

onde, $\mathrm{k}$ e $\mathrm{n}$ são as constantes que definem a viscosidade do fluido, nesse caso ela não pode ser calculada diretamente como no modelo de Bingham.

AZEREDO [14] mostrou que as pastas de cal-metacaulim seguem o modelo quase perfeitamente de fluidos de HB, ou seja, onde a viscosidade não pode ser obtida diretamente como no modelo de Bingham.

Da literatura é conhecido que o comportamento reológico de concretos e argamassas é muito influenciado pela sua pasta no estado fresco. Com isso, vários estudos vêm sendo realizados sobre a determinação dos parâmetros reológicos destas pastas a fim de entender melhor o comportamento desses materiais.

CARDOSO et al. [15] mostraram que o estudo do comportamento das argamassas no estado fresco viabiliza uma moldagem adequada, além de possibilitar minimizar a ocorrência de manifestações patológicas após o endurecimento dos revestimentos, como fissuração, desplacamento e eflorescências.

SAAK et al. [16] afirmam que a principal propriedade do concreto fresco é a sua trabalhabilidade, debatida em várias décadas por cientistas e engenheiros. Esta propriedade é resultante da consistência, fluidez e compacidade.

BANFILL [17] mostrou que ao confrontar os parâmetros reológicos da pasta de cimento com os do concreto, não se encontrará relação direta. E que há uma tendência de comportamento reológico de materiais com base cimentícia, que variam segundo a presença dos agregados.

QUARCIONI [18] mostrou que o estudo em pastas cimentícias é de grande valia quando se quer avaliar o comportamento do material durante seu manuseio e aplicação.

Muitos trabalhos também têm analisado o comportamento reológico de pastas cimentícias contendo adições pozolânicas, em todas elas as curvas de fluxo obtidas se ajustaram melhor ao modelo de HerschelBulkley e as adições contribuíram para um melhor escoamento e menor viscosidade da mistura [19, 20, 21, 22 e 23].

BANFILL e FRÍAS [19] mostraram que pastas de cimento Portland contendo metacaulim comercial apresentaram uma menor tensão de escoamento quando comparadas com pastas contendo resíduo da indústria de papel, sendo esse comportamento associado à forma das partículas do metacaulim, que são lamelares.

JANOTKA et al. [21] mostraram que, para as pastas de cimento Porland contendo até $40 \%$ de metacaulim e avaliadas no estado fresco, com aumento do teor de metacaulim a tensão de escoamento também aumentou, o que ocorreu devido à alta área específica do metacaulim utilizado, fazendo com que os grãos ficassem mais aglomerados.

VANCE et al. [12] avaliaram misturas binárias e ternárias contendo vários tipos de adições, e naquelas contendo metacaulim houve diminuição da tensão de escoamento e viscosidade. Os autores explicam que nas misturas com o metacaulim ocorre um menor empacotamento das partículas. Além disso, eles atribuem este fato também ao maior teor de água e o espaço interpartícula.

TCHAMDJOU et al. [25] mostraram que as misturas contendo até 45\% de uma pozolana natural, em substituição ao cimento Portland, apresentaram decréscimo tanto na tensão de escoamento como na viscosidade. Esse fato estaria relacionado ao mau empacotamento das partículas na mistura, fazendo com que elas sejam mais fáceis de escoar.

SHANAHAN et al. [26] mostraram que a tensão de escoamento diminuiu quando se usou metacaulim e/ou sílica ativa e aumentou quando se usou escória e cinza volante. A razão para isto foi devido à alta área específica desses materiais. $\mathrm{O}$ mesmo efeito ocorreu para a viscosidade aparente.

Todos esses trabalhos têm mostrado que uso de adições pozolânicas em pastas cimentícias contribuem de alguma forma para melhorar seu comportamento reológico, do ponto de vista de facilitar seu manuseio e mistura no estado fresco. O uso de adições, oriundas ou não de resíduos, tem sido cada vez mais investigado 
como também aplicado na prática da construção. Dentre esses, podem-se destacar aqueles provenientes de resíduos de tijolos cerâmicos moídos. O resíduo de tijolo cerâmico moído vem sendo bastante estudado na literatura, onde já se comprovou seu potencial como material pozolânico em argamassas e concretos, após passar por um processo de beneficiamento [27, 28 e 29], além de apresentar uma alternativa para a problemática da gestão de resíduos. Porém, pouco se sabe sobre sua influência no comportamento reológico de argamassas e concretos.

Este trabalho, então, busca analisar a influência do resíduo de tijolo cerâmico moído (RTM) e do metacaulim (MC) no comportamento reológico de pastas cimentícias, utilizando o método de reometria de fluxo.

\section{MATERIAIS E MÉTODOS}

\subsection{Materiais}

Neste trabalho utilizou-se o cimento CP V-ARI RS, classificado como cimento Portland de alta resistência inicial resistentes à sulfatos de acordo com a norma NBR 5737 [30], obtido no comércio local. Também foram utilizados um MC comercial e um RTM obtido em laboratório.

Para obtenção do RTM foi realizada uma coleta manual em uma loja de materiais de construção local, onde procurou-se selecionar os blocos cerâmicos que apresentassem coloração uniforme, a fim de se obter uma amostra homogênea do material. Esse material foi encaminhado ao laboratório para realizar o seu beneficiamento, que se deu através de fragmentação e moagem. A fragmentação foi realizada em um britador de mandíbulas modelo $\mathrm{J} / 58$, fabricado pela PLANGG, com intuito de reduzir os blocos em pedaços menores. Após a fragmentação, colocou-se o material em um moinho de bolas modelo SONNEX I-4205, fabricado pela CONTENCO, com capacidade de $50 \mathrm{dm}^{3}$. Na moagem foram usadas 30.000 rotações, pois conforme estudo realizado por CARVALHO [27], o gasto energético realizado para um número maior de rotações não traria ganhos consideráveis para a finura do material (Figura 1).

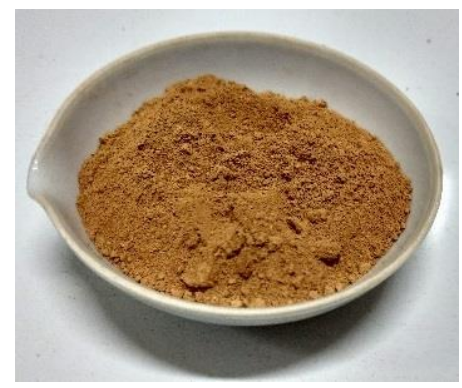

Figura 1: Amostra do resíduo de tijolo cerâmico moído após o seu beneficiamento.

\subsection{Caracterização dos materiais}

Os materiais foram caracterizados quanto às suas propriedades físicas, químicas e mineralógicas. No que diz respeito às propriedades físicas, foram realizados ensaios de massa unitária, massa específica [31 e 32] e granulometria a laser. Na determinação da massa específica foram adotados os procedimentos descritos na NBR NM 23 [31]. A massa unitária foi determinada no estado solto utilizando um recipiente de massa e volume conhecidos, no qual se fez três leituras para cada material e a média dessas leituras foi o valor considerado. Na Tabela 1 são apresentados os dados referentes a essas características.

Analisando a tabela 1, percebe-se que o MC apresenta uma massa unitária quase três vezes menor em relação ao RTM. No entanto, com relação à massa específica, os dois materiais possuem valores similares. A semelhança na massa específica pode ser explicada pela natureza dos materiais, visto que, ambos são de origem de solo caulinítico. Já a diferença em relação à massa unitária pode ser explicada devido ao processo de obtenção utilizado para cada material, como a moagem e queima.

Tabela 1: Características físicas das adições pozolânicas.

\begin{tabular}{l|l|l}
\hline MATERIAL & MASSA ESPECÍFICA $\left(\mathbf{g} / \mathbf{c m}^{3}\right)$ & MASSA UNITÁRIA $\left(\mathbf{g} / \mathbf{c m}^{3}\right)$ \\
\hline RTM & 2,64 & 1,35 \\
\hline
\end{tabular}




\begin{tabular}{l|l|l}
\hline $\mathrm{MC}$ & 2,62 & 0,41 \\
\hline
\end{tabular}

A granulometria a laser foi realizada usando um equipamento do tipo CILAS, modelo 1064, em uma faixa de tamanho entre $40 \mathrm{~nm}$ e $500 \mu \mathrm{m}$ de diâmetro. A amostra foi dispersa no próprio equipamento em uma cuba de $400 \mathrm{ml}$ de água destilada sob ação de um agitador mecânico por 20 minutos, essa cuba dispunha de ultrassom que opera a frequência de $55 \mathrm{~Hz}$ e amplitude de $55 \%$. Os resultados estão expressos em curvas de distribuição granulométrica das partículas dos materiais finos e também em um histograma, com o volume dessas partículas (Figura 2).
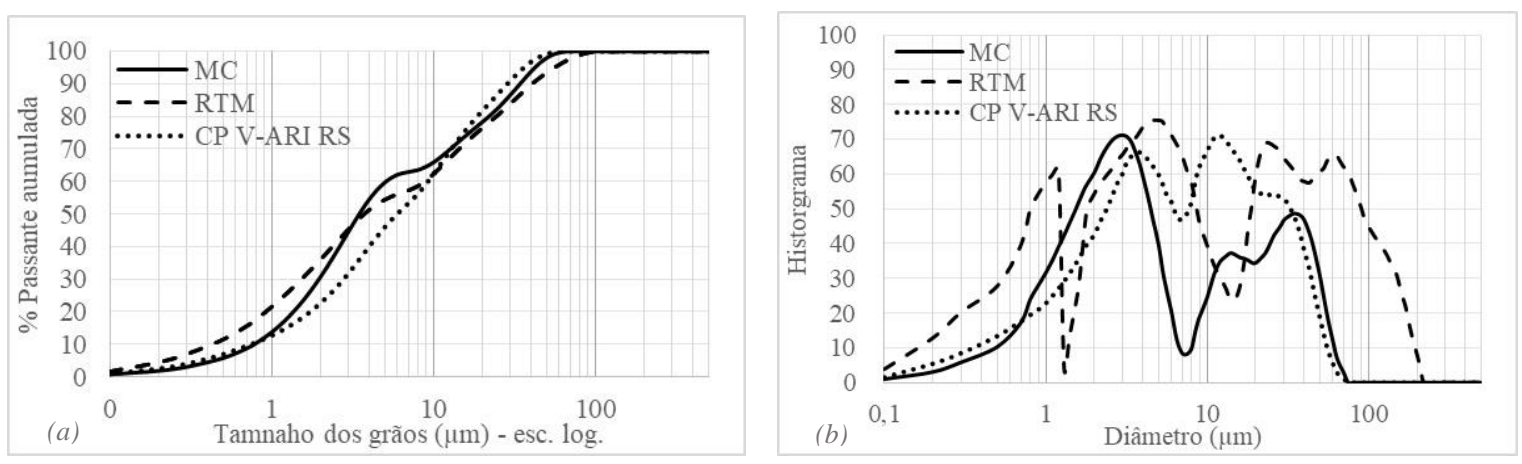

Figura 2: (a) Curva granulométrica dos materiais finos; (b) Volume das partículas dos materiais finos.

Segundo a análise granulométrica, o MC apresenta diâmetro médio de 11,07 $\mu \mathrm{m} ; 10 \%$ dos sólidos acumulados são menores que $0,78 \mu \mathrm{m} ; 50 \%$ são menores que $3,48 \mu \mathrm{m}$ e $90 \%$ são menores que $34,35 \mu \mathrm{m}$. Para o RTM o diâmetro médio é de $13,47 \mu \mathrm{m} ; 10 \%$ dos sólidos acumulados são menores que $0,42 \mu \mathrm{m} ; 50 \%$ são menores que 3,74 $\mu \mathrm{m}$ e $90 \%$ são menores que 40,77 $\mu \mathrm{m}$. CARVALHO [27] mostrou que o diâmetro médio do RTM é responsável pelo efeito fíler e pela geração de pontos de nucleação. Com relação ao CP V-ARI RS o seu diâmetro médio é de $10,77 \mu \mathrm{m} ; 10 \%$ dos sólidos acumulados são menores que $0,75 \mu \mathrm{m} ; 50 \%$ são menores $5,96 \mu \mathrm{m}$ e $90 \%$ são menores que $28,67 \mu \mathrm{m}$. Ainda é possível ver que a maior concentração de partículas dos três materiais acontece entre 1 e $100 \mu \mathrm{m}$.

$\mathrm{O}$ cimento apresenta curva granulométrica bem graduada, enquanto que para o MC a curva apresenta pequena descontinuidade. A curva do RTM é semelhante a do metacaulim, porém com menor descontinuidade. Como a curva do cimento está abaixo das demais, no intervalo entre $1 \mu \mathrm{m}$ e $10 \mu \mathrm{m}$, pode-se dizer que o mesmo possui porcentagem menor de partículas neste intervalo. Já o metacaulim apresenta maior quantidade de grãos entre a faixa de $0 \mu \mathrm{m}$ e $1 \mu \mathrm{m}$. Em relação ao histograma é possível perceber que no intervalo entre $0,6 \mu \mathrm{m}$ e $1,12 \mu \mathrm{m}$ existe maior quantidade de grãos de resíduo de tijolo cerâmico moído. Entre 1,5 $\mu \mathrm{m}$ e 3,0 $\mu \mathrm{m}$ ocorre maior concentração de partículas de MC.

A composição química dos materiais finos foi determinada de forma semiquantitativa por espectrômetro de fluorescência de raios-X em um equipamento SHIMADZU, modelo XRF-1800. Na Tabela 2 são apresentados os dados obtidos dessa análise química.

Tabela 2: Composição química dos materiais finos (fluorescência de raio-x).

\begin{tabular}{l|l|l|l|l|l|l|l|l}
\hline$\%$ & $\mathrm{SiO}_{2}$ & $\mathbf{A l}_{2} \mathbf{O}_{3}$ & $\mathrm{Fe}_{2} \mathbf{O}_{3}$ & $\mathbf{K}_{2} \mathbf{O}$ & $\mathbf{M g O}$ & $\mathbf{C a O}$ & $\mathrm{TiO}_{2}$ & OUTROS \\
\hline RTM & 68,902 & 12,420 & 8,405 & 3,415 & 2,057 & 1,534 & 1,344 & 0,525 \\
\hline MC & 67,658 & 24,387 & 4,849 & 0,794 & 0,356 & 0,307 & 1,101 & 1,343 \\
\hline CPV-ARI RS & 22,601 & 3,480 & 3,006 & 0,932 & 1,731 & 62,068 & 0,247 & 1,214 \\
\hline
\end{tabular}

Com base na Tabela 2 é possível observar que o MC é constituído por cerca de $67,7 \%$ de $\mathrm{SiO}_{2}$ e $24,4 \%$ de $\mathrm{Al}_{2} \mathrm{O}_{3}$. A soma da porcentagem desses dois óxidos resulta em um valor maior que $70 \%$, o que dar a entender que o MC, no que diz respeito à sua composição química, obedece aos critérios da NBR 12653 [33] e pode ser considerado material pozolânico. Para o RTM, a soma dos óxidos de ferro, alumínio e sílica são maiores que 70\%, como determina a NBR 12653 [34], para materiais pozolânicos. Também é possível perceber que o RTM possui maior teor de $\mathrm{Fe}_{2} \mathrm{O}_{3}$ que o MC, o que explica sua coloração mais escura. $\mathrm{O}$ teor de 
$\mathrm{C}_{3} \mathrm{~A}$ para o $\mathrm{CP} \mathrm{V}$-ARI RS foi menor que $8 \%$, o que implica dizer que o cimento está de acordo com a norma NBR 5737 [30].

A caracterização por difração de raios-x foi realizada pelo equipamento Siemens Bruker, modelo D5000, sob as seguintes condições de ensaio: radiação $\mathrm{CuK} \alpha$ de comprimento de onda $\lambda=1.5418 \AA$ com raios-X em $40 \mathrm{kv}$ e $30 \mathrm{~mA}$, velocidade de leitura de $1^{\circ} / \mathrm{min}$ num intervalo de $5^{\circ}$ a $70^{\circ} 2 \Theta$ a um passo angular de $0,02^{\circ}$ 2Ө. A identificação dos picos foi feita pelo software MDI JADE 5.0.

Na Figura 3 são mostrados os difratogramas do RTM e do MC, onde nota-se que o RTM apresenta picos mais intensos que o MC, também é possível observar que há um halo amorfo entre os ângulos $2 \Theta^{\circ} 20^{\circ}$ $30^{\circ}$ bem mais evidente no MC que no RTM. Dessa forma, o RTM pode ser considerado menos amorfo que o MC. Os principais elementos encontrados do DRX foram ililta, caulinita e quartzo para ambos os materiais.

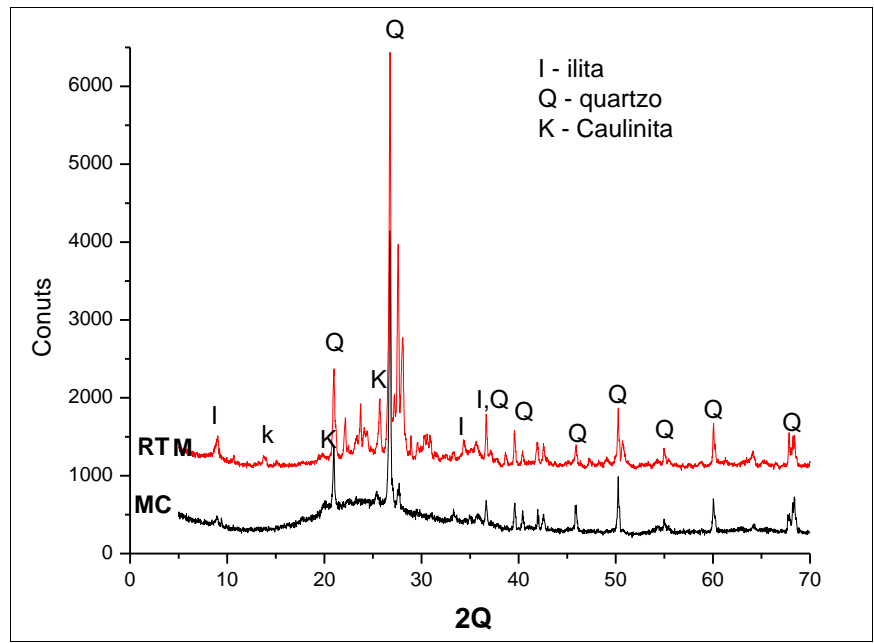

Figura 3: Difração de raio-x do resíduo de tijolo cerâmico moído e do metacaulim.

\subsection{Composição das misturas e preparação das pastas}

As misturas das pastas foram avaliadas em seu estado fresco para diferentes relações água/aglomerante contendo variações de proporções de $1: 0,2 ; 1: 0,5 ; 1: 0,8$, em massa, de cimento Portland e metacaulim, como também de cimento Portland e RTM. Ao todo foram estudadas 10 misturas de pastas, sendo 5 utilizando RTM e 5 utilizando metacaulim. Na Tabela 3 estão descritas as composições das misturas de forma mais detalhada.

Tabela 3: Composições das misturas estudadas.

\begin{tabular}{|c|c|c|c|c|c|}
\hline GRUPO & PASTA & CIMENTO (g) & $M C(g)$ & RTM (g) & ÁGUA (g) \\
\hline \multirow{5}{*}{ A } & $1 \mathrm{~A}$ & 50 & 10 & - & 30 \\
\hline & $2 \mathrm{~A}$ & 50 & 10 & - & 42 \\
\hline & $3 \mathrm{~A}$ & 50 & 40 & - & 50 \\
\hline & $4 \mathrm{~A}$ & 50 & 40 & - & 63 \\
\hline & $5 \mathrm{~A}$ & 50 & 25 & - & 45 \\
\hline \multirow{5}{*}{ B } & $1 \mathrm{~B}$ & 50 & - & 10 & 30 \\
\hline & 2B & 50 & - & 10 & 42 \\
\hline & $3 \mathrm{~B}$ & 50 & - & 40 & 50 \\
\hline & 4B & 50 & - & 40 & 63 \\
\hline & $5 \mathrm{~B}$ & 50 & - & 25 & 45 \\
\hline
\end{tabular}

Inicialmente pesou-se a quantidade de água, cimento e pozolanas (MC e RTM) separadamente numa balança eletrônica digital, com precisão de 0,001g. Logo em seguida, para a preparação das pastas, os finos foram misturados manualmente. Por fim, foi adicionado água e realizada a mistura manual da pasta, durante 
2 minutos, para obter uma pasta homogênea.

Os ensaios foram realizados em 4 tempos diferentes de hidratação (0, 20, 40 e 60 min), com base no trabalho de SILVA [35], contados a partir do contato da mistura seca com a água. Foi utilizado o reômetro rotacional de placas paralelas Rheotest RN/PR, com diâmetro das placas de $40 \mathrm{~mm}$ e espaçamento entre elas de $1 \mathrm{~mm}$. A aquisição de dados foi feita utilizando uma frequência de coleta de um ponto a cada 10 segundos. Para evitar o deslizamento entre as placas e a pasta foi utilizado um revestimento texturizado nas placas (Figura 4), conforme os trabalhos de BETIOLI et al. [9], TEIXEIRA et al. [36] e MEDEIROS et al. [37].

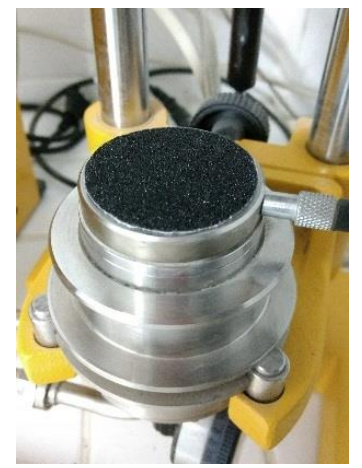

Figura 4: Detalhe do revestimento texturizado utilizado para melhorar a aderência entre as pastas cimentícias e as placas do reômetro.

Os ensaios foram do tipo rampa, que consistem na aplicação de taxa crescente de cisalhamento com aceleração de 0 a $100 \mathrm{~s}^{-1}$ em 2 min, seguida de desaceleração até 0 , em mais 2 min [9, 36 e 37$]$.

\section{RESULTADOS E DISCUSSÕES}

Na Figura 5 são apresentados os resultados das curvas de tensão de cisalhamento versus taxa de cisalhamento para cada tempo de hidratação das misturas do grupo A e B. Todas as pastas necessitaram de tensão de cisalhamento inicial para que o escoamento ocorresse.

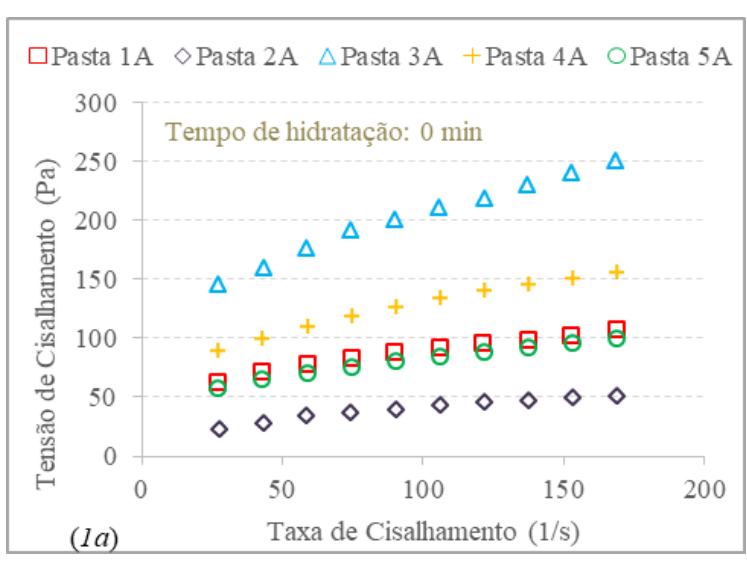

$\square$ Pasta $1 \mathrm{~A} \diamond$ Pasta $2 \mathrm{~A} \triangle$ Pasta $3 \mathrm{~A}+$ Pasta $4 \mathrm{~A}$ OPasta $5 \mathrm{~A}$

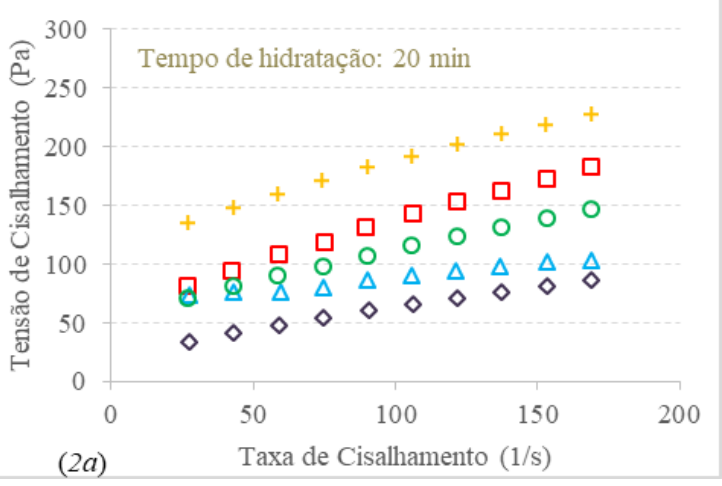

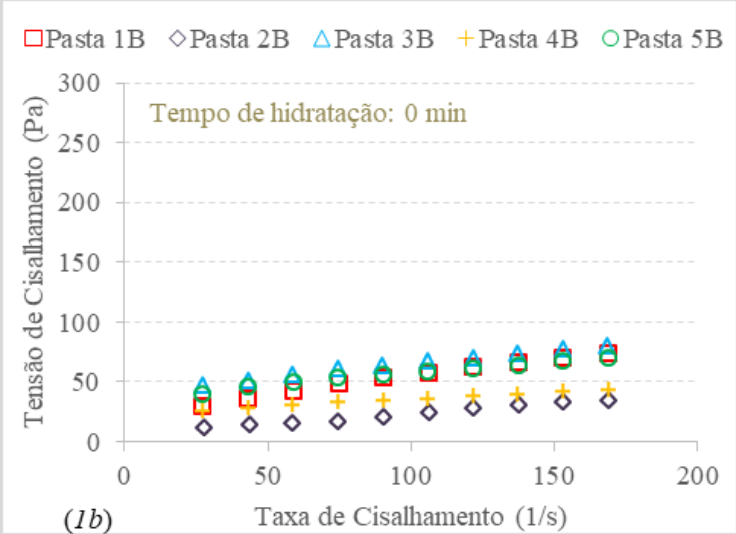

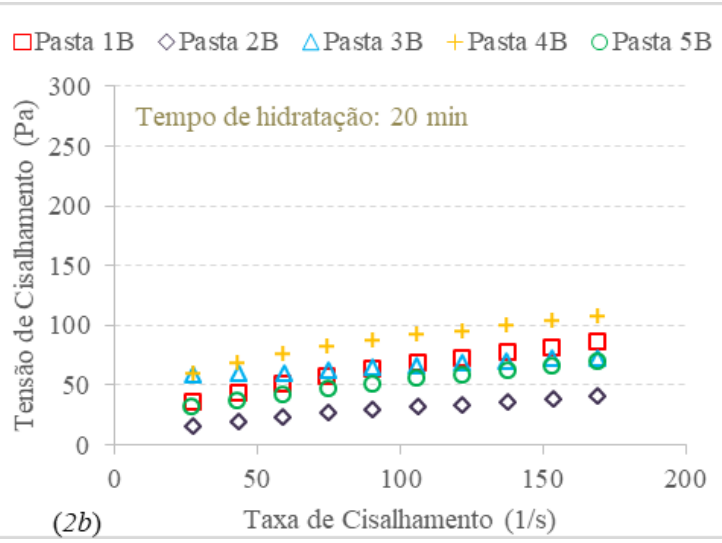



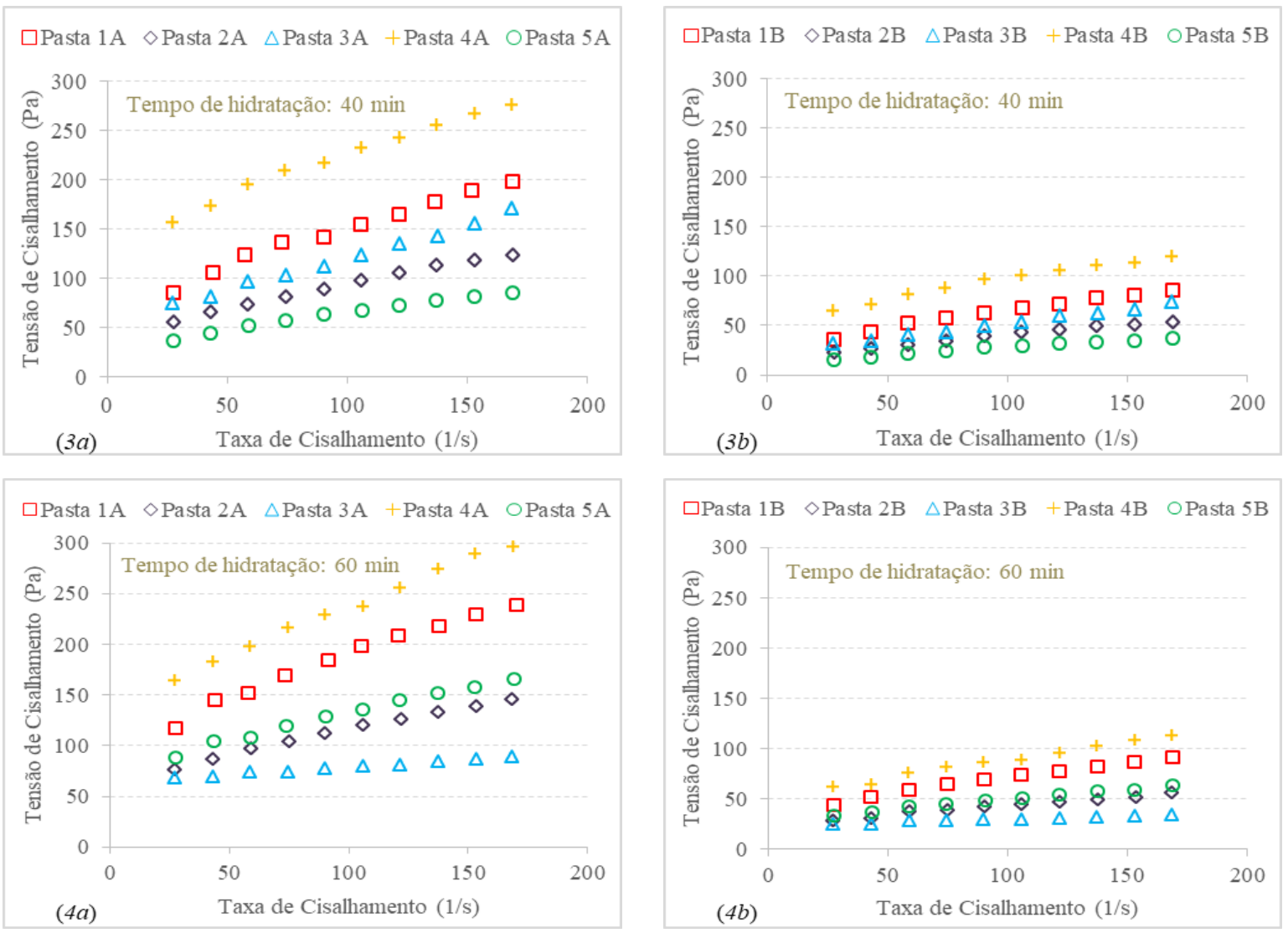

Figura 5: Tensão de cisalhamento versus taxa de cisalhamento - (a) misturas contendo metacaulim; (b) misturas contendo resíduo de tijolo cerâmico moído.

Ao analisar as curvas, nota-se que todas apresentam um comportamento não linear, mostrando uma tendência para um modelo de HB, que também pode ser conhecido como um modelo de Bingham generalizado, como citado por TEXEIRA et al. [36]. Esse comportamento também foi encontrado em outros estudos que abordavam pastas cimentícias contendo adições [19, 21, 22, 36, 38 e 39].

De modo geral, observa-se que as pastas contendo resíduo de tijolo cerâmico moído apresentaram menores valores de tensão de escoamento inicial (de $20 \mathrm{~Pa}$ à $60 \mathrm{~Pa}$ ) que aquelas contendo metacaulim (atingindo até $150 \mathrm{~Pa}$ ), ou seja, maior facilidade para iniciar o escoamento. Esse fato pode ser explicado devido ao menor volume de partículas presente nas misturas contendo RTM, uma vez que a sua massa unitária é bem maior que a do MC. O menor volume de partículas contribui melhorando o escoamento da pasta, pois as partículas encontram-se mais separadas umas das outras, ou seja, estão dispersas na mistura. Já nas pastas com alto volume de concentração de partículas, houve aglomeração das mesmas, ocasionando maiores valores de tensão de escoamento, como é o caso das pastas contendo MC. Fatores como a forma e mineralogia do grão, as forças inter-partículas, a rugosidade da partícula, entre outros [39 e 40], também podem influenciar na tensão de escoamento.

Com relação ao tempo de hidratação observa-se que todas as pastas tenderam a aumentar a tensão de escoamento quanto maior foi esse tempo. Porém, o mesmo não aconteceu para a pasta 3, em ambos grupos de misturas (A e B), ou seja, aquelas que contém maior teor de finos e menor teor de água, a tensão diminuiu ao longo do tempo. Isso pode ser explicado pelo fato dessas pastas terem mais partículas de adição pozolânica que de cimento, o que contribui para o aumento do tempo de pega, fazendo com que estas misturas ainda apresentassem facilidade de escoamento, mesmo em 60 minutos. As pastas contendo até $50 \%$ de metacaulim (pastas 2A e 5A) foram as que apresentaram menor tensão de escoamento ao longo do tempo, comparando com as demais pastas contendo metacaulim. Essa diminuição da tensão de escoamento também ocorreu para as pastas estudadas nos trabalhos de VANCE et al. [12], SKRIPKIŪNAS et al. [24], TCHAMDJOU et al. [25] e SHANAHAN et al. [26], onde a razão atribuída para isso foi o mau empacotamento das partículas, bem como uma maior demanda de água nas misturas.

Nas pastas contendo RTM, o comportamento das curvas de tensão de escoamento foi similar ao longo 
do tempo, ou seja, até 60 minutos não houve influência que ocasionasse alteração no escoamento. Isto pode ser atribuído ao fato de que as partículas de RTM não se aglomeraram ao longo do tempo ou formaram uma estrutura bem conectada tridimensionalmente com as partículas de cimento devido a algum processo de hidratação, já que nessas misturas existe um menor volume de partículas.

De modo geral, para todas as pastas, ainda é possível observar que o aumento da quantidade de água ocasionou a diminuição da tensão de escoamento quando comparando as misturas com mesma quantidade de materiais finos. O aumento de água torna a mistura mais dispersa, fazendo com que a tensão de escoamento diminua.

Na Figura 6 são apresentados os gráficos referentes à viscosidade versus taxa de cisalhamento das pastas contendo MC (grupo A) ou RTM (grupo B).
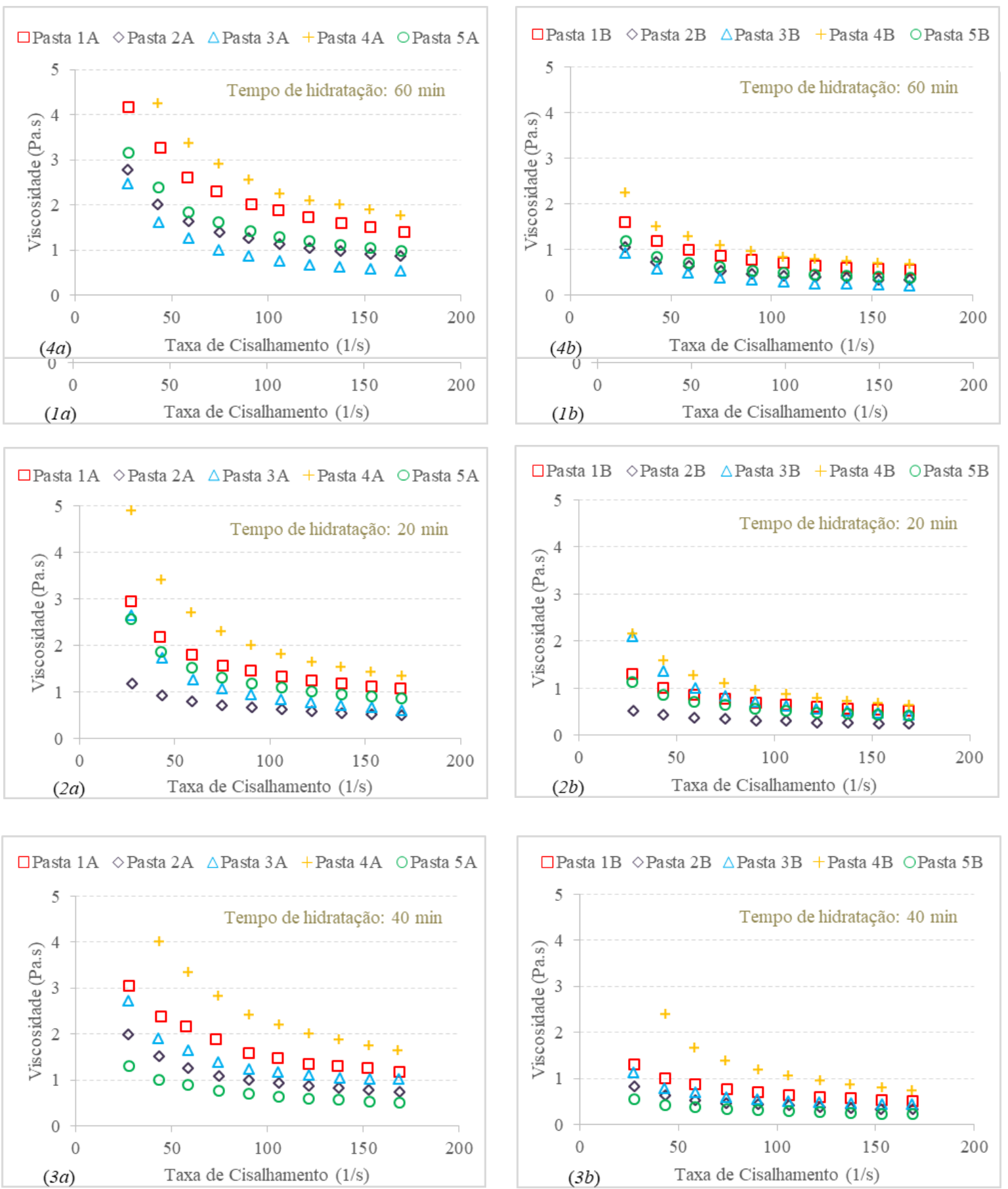

Figura 6: Viscosidade versus taxa de cisalhamento - (a) misturas contendo metacaulim; $(b)$ misturas contendo resíduo de tijolo cerâmico moído. 
Ao analisar a Figura 6 é possível perceber que em todas as pastas a viscosidade aparente, obtida através da relação entre a tensão de cisalhamento e a taxa de cisalhamento, diminuiu com o aumento da taxa de cisalhamento, caracterizando a natureza tixotrópica do material. Este fato também é observado nos trabalhos de BETIOLI et al. [9] e TEIXEIRa et al. [36]. Nas pastas contendo MC a viscosidade alcançou valores da ordem de 4 Pa.s, enquanto que para aquelas contendo RTM foi na ordem de 2 Pa.s.

Comparando as pastas dos dois grupos é possível notar que todas as pastas contendo RTM (grupo B) apresentaram menor viscosidade aparente que aquelas contendo MC (grupo A). Esse comportamento pode ser justificado pelo fato dos grãos do RTM apresentarem maior massa unitária, o que faz com que essas pastas tenham menor volume de grãos comparadas aquelas com MC, ocasionando menos aglomerados na mistura, devido menor quantidade de finos nas pastas. Esse efeito está diretamente relacionado ao volume de partículas em suspensão, quanto maior for esse volume maior também será a viscosidade da mistura [41 e 42].

Outro fator que influencia na viscosidade é o formato do grão, suspensões com alto volume de grãos não esféricos concentrado a viscosidade aumenta, pois esses grãos poderão estabelecer ligações entre si formando um reticulado mais ou menos rígido que prenderá a água e aumentará a viscosidade [41 e 43]. De um modo geral as pastas apresentaram um leve aumento da viscosidade com o aumento do tempo de hidratação.

\section{CONCLUSÕES}

Este trabalho teve como principal objetivo avaliar o comportamento de dois tipos de adições pozolânicas na reologia de pastas cimentícias, usando um reômetro de placas paralelas.

O resíduo de tijolo cerâmico moído (RTM) e o metacaulim (MC) apresentaram características químicas e mineralógicas semelhantes, entretanto, a massa unitária do RTM foi até 3 vezes maior que a do MC, o que implica dizer que as pastas com MC tiveram maior concentração de volume de sólidos.

Todas as pastas apresentaram comportamento Binghaminiano, necessitando de uma tensão de escoamento inicial para haver escoamento, além de se mostrarem com tendência para o modelo de HerschelBulkley.

As pastas contendo MC apresentaram maiores tensões de escoamento e viscosidade quando comparadas aquelas contendo RTM. Esse fato está diretamente ligado a maior concentração de sólidos nas pastas com metacaulim, o que provoca uma maior dificuldade de escoamento, devido à aglomeração das partículas nessas misturas. Uma maior quantidade de água também contribuiu para diminuição da tensão de escoamento e da viscosidade quando comparando as pastas com mesma quantidade de finos.

Com relação ao comportamento dessas pastas em função do tempo, observa-se que, de modo geral, todas as pastas apresentaram tendência de aumento da tensão de escoamento e viscosidade quanto maior fosse o tempo de hidratação. Porém, foi observado que as pastas com $50 \%$ de MC não sofreram aumento na tensão de escoamento nem na viscosidade, comparando os tempos de 0 e 60 minutos, onde houve diminuição desses valores para o maior tempo de hidratação. Esse fato pode ter ocorrido devido à grande quantidade de MC na mistura, que contribuiu para um retardamento da pega da pasta cimentícia e também para um mau empacotamento das partículas na mistura, além da maior quantidade de água.

\section{AGRADECIMENTOS}

Os autores agradecem ao CNPq pelo financiamento desta pesquisa, bem como ao Laboratório de Ensaios de Materiais e Estruturas (LABEME) do Centro de Tecnologia da Universidade Federal da Paraíba pelo apoio técnico e por ceder o uso de seus equipamentos para realização dos ensaios. Também ao Instituto Federal de Educação, Ciência e Tecnologia da Paraíba no apoio através do uso de alguns equipamentos.

\section{BIBLIOGRAFIA}

[1] CASTRO A. L., LIBÓRIO J. B. L., PANDOLFELLI V. C., "Reologia de concretos de alto desempenho aplicados na construção civil”, Revista Cerâmica, v. 57, pp. 63-75, 2011.

[2] BANFILL, P. F. G., "Rheological methods for assessing the flow properties of mortar and related materials", Construction and Building Materials, v. 8, pp. 43-50, 1994.

[3] MACHADO, J.C.V., Reologia e escoamento de fluidos: ênfase na indústria do petróleo, Ed. Interciência: PETROBRÁS, Rio de Janeiro, 2002.

[4] SATO, V. Y., EVANGELISTA, K. T. C., TEIXEIRA, J. E. S. L., et al., "Estudo das propriedades reológicas de pastas de cimento de concretos bombeados", In: Congresso Brasileiro do Concreto, 54., Anais, Maceió: IBRACON, 2012. 
[5] LISBÔA, E. M., "Obtenção do concreto auto-adensável utilizando resíduo do beneficiamento do mármore e granito e estudo de propriedades mecânicas", Dissertação de M. Sc., PPGEC/UFAL, Maceió, AL, Brasil, 2004.

[6] CAVALCANTI, D. J. H., "Contribuição ao estudo de propriedades do concreto auto-adensável visando sua aplicação em elementos estruturais”, Dissertação de M. Sc., PPGEC/UFAL, Maceió, AL, Brasil, 2006.

[7] VENÂNCIO, P. B. F., "Dosagem de argamassa para estaca raiz", Dissertação de M. Sc., PECC/UNB, Brasília, DF, Brasil, 2008.

[8] SAAK, A. W., JENNINGS, H. M., SHAH, S. P., "The Influence of Wall Slip on Yield Stress and Viscoelastic Measurements of Cement Paste", Cement and Concrete Research, v. 31, n. 2, pp. 205-212, fev. 2001.

[9] BETIOLI, A. M., JOHN, V. M., GLEIZE, P. J. P., et al., "Rheological characterization of cement paste: association of complementary techniques", Ambiente Construído , v.9, n.4, pp. 37-48, 2009.

[10] STRUBLE, L. J., LEI, W-G., "Rheological Changes Associated with setting on cement paste", Advance Cement Based Materials, n.2, pp. 224-230, 1995.

[11] AZEREDO, G. A., MOREL, J.C., "Applicability of rheometers to characterizing earth mortar behavior. Part I: experimental device and validation", Materials and Structure, v. 41, pp. 1465-1472, 2008.

[12] VENCE, K., KUMAR, A., SANT, G., et al., "The rheological properties of ternary binders containing Portland cement, limestone, and metakaolin or fly ash.”, Cement and Concrete Research, v. 52, pp. 196-207, 2013.

[13] FERRARIS, C., LARRAD, F., MARTYS, N., "Fresh Concrete Rheology: Recent Developments", Natl Inst. Stand Thecnol, pp. 215-241, 2001.

[14] AZEREDO, A. F. N., "Estudo do resíduo de caulim em argamassas a base de cal quanto às suas propriedades frescas, endurecidas e microestruturais", Tese de D. Sc., POSCIVIL/UFPE, Recife, PE, Brasil, 2012.

[15] CARDOSO, F. A., PILEGGI, R. G., JOHN, V. M., "Rheological behavior of mortars under different squeezing rates", Cement and Concrete Research, v.39, pp.748-775, 2009.

[16] SAAK, A. W., JENNINGS, H. M., SHAH, S. P. "A generalized approach for the determination of yield stress by slump and slump flow", Northwestern University; Evanston, IL, USA. 2004.

[17] BANFILL, P. F. G., "The Rheology of Fresh Cement and Concrete - A Review", In: 11th International Cement Chemistry Congress, pp. 61-130, May, 2003.

[18] QUARCIONI, V. A. N. J. D., "Estudo da cal hidratada nas idades iniciais da hidratação do cimento Portland", Tese de D. Sc., PPGEC/USP, São Paulo, SP, Brasil, 2008.

[19] BANFILL, P. G. F., FRÍAS, M., "Rheology and conduction calorimetry of cement modified with calcined paper sludge", Cement and Concrete Research, v. 37, pp. 184-190, 2007.

[20] BANFILL, P. F. G., RODRÍGUEZ, O., ROJAS, S., et al., "Effect of activation conditions of kaolinite waste on rheology of blended cement pastes", Cement and Concrete Research, v. 39, pp. 843-848, 2009.

[21] JANOTKA, I., PUERTAS, F., PALACIOS, M., et al., "Metakaolin sand-blend-cement pastes: Rheology, hydration process and mechanical properties", Construction and Building Materials, v.24, pp. 791-802, 2009.

[22] MANSOUR, S. M., ABADILA, M. T., BEKKOUR, K., et al., "Improvement of rheological behavior of cement pastes incorporating metakaolin", European Journal of Scientific Research, v 42, pp. 442-452, 2010.

[23] SAFI, B., BENMOUNAH, A., SAIDI, M., "Rheology and zeta potencial of cement pastes containing calcined sitl and ground granulated blast-furnace slag", Materiales de Construcción, v. 61, pp. 353- 370, 2011.

[24] SKRIPKIŪNAS, G., MACIJAUSKAS, M., NAGROCKIENĖ, D., et al., "The influence of biomass fly ash on the plasticizing effects in cement pastes", Procedia Engineering, v. 172, pp.1015-1022, 2017.

[25] TCHAMDJOU, W. H. J., CHERRADI, T., ABIDIA, M. L., et al., Influence of different amounts of natural pozzolan from volcanic scoria on the rheological properties of portland cement pastes", Energy Procedia, v.139, pp. 696-702, 2017.

[26] SHANAHAN, N., TRAN, V., WILLIAMS, A., et al., "Effect of SCM combinations on paste rheology and its relationship to particle characteristics of the mixture", Construction and Building Materials, v. 123, pp. 745-753, 2016. 
[27] CARVALHO, C. M., "Caracterização de resíduos da indústria cerâmica e seu emprego em argamassas de cimento Portland”, Dissertação de M. Sc., PPGEM/UFPB, João Pessoa, PB, Brasil, 2016.

[28] VIEIRA, A. A. P., "Estudo do aproveitamento de resíduos de cerâmica vermelha como substituição pozolânicas em argamassa e concreto", Dissertação de M. Sc., PPGECAM/UFPB, João Pessoa, PB, Brasil, 2005.

[29] JERÔNIMO, V.L., "Estudo de concreto com adição de resíduo da indústria de cerâmica vermelha com foco na corrosão de armadura", Tese de D. Sc., PPGEC/UFRGS, Porto Alegre, RS, 2014.

[30] ASSOCIAÇÃO BRASILEIRA DE NORMAS TÉCNICAS - ABNT, NBR 5737: Cimento Portland resistente a sulfatos, Rio de Janeiro, 1992.

[31] _ _ NBR NM 23: Cimento Portland e outros materiais em pó - Determinação da massa específica, Rio de Janeiro, 2001.

[32] 2009. NBR NM 52: Agregado graúdo - Determinação de massa específica e massa específica aparente,

[33] .NBR 12653: Materiais pozolânicos: requisitos, Rio de Janeiro, 2012. .NBR 12653: Materiais pozolânicos: requisitos, Rio de Janeiro, 2012b.

[35] SILVA, D. A. "Efeitos dos polímeros HEC e EVA na microestrutura de pastas de cimento Portland", Tese de D. Sc., Departamento de Engenharia dos Materiais/UFSC, Florianópolis, SC, Brasil, 2001.

[36] TEIXEIRA, J. E. S. L., SATO, V. Y., AZOLIN, L. G., et al., "Study of cement pastes rheological behavior using dynamic shear rheometer", Ibracon, v.7, 6, pp. 922-939, 2014.

[37] MEDEIROS, G. A., AZERÊDO, A.F.N., AZERÊDO, G. A. Anais do 59 Congresso Brasileiro do Concreto, Bento Gonçalves, RS, 2017.

[38] MANSOUR, S. M., ABADILA, M. T., BEKKOUR, K., et al., "Improvement of rheological behavior of cement pastes incorporating metakaolin”, European Journal of Scientific Research, v. 42, pp. 442-452, 2010.

[39] PEDRAJAS, C., RAHHAL, V., TALERO, R., "Determination of characteristic rheological parameters in Portland cement pastes", Construction and Building Materials, v.51, pp. 484-491, 2014.

[40] KASHANI, A., NICOLAS, R.S., QIAO, G.G., et al., "Modelling the yield stress cement-slag-fly ash pastes based on particle size distribution", Powder Technology, v. 266, pp. 203-209, 2014.

[41] BARNES, H. A., HITTON, J. F., WALTERS, F. R. S. An introduction to reheology. 1ed., Elsevier, Amsterdan, 1989.

[42] TRADOR, T. F., Rheology of dispersions: Principles and applications., ed. Wiley-VCH, United Kingdon, 2010.

[43] SANTOS, P. S., Ciência e Tecnologia de argilas, 2 ed. rev. amp., São Paulo, Edgard Blucher, 1992.

\section{ORCID}

João Sérgio Filho

Kássia Sinhorelli

Guilherme Medeiros

Aline Azeredo

Givanildo Azeredo https://orcid.org/0000-0003-2747-3572

https://orcid.org/0000-0003-3560-3349

https://orcid.org/0000-0002-4302-3822

https://orcid.org/0000-0002-2327-8780

https://orcid.org/0000-0001-6572-8287 